(leucocytes) que les couches plus éloignées, disposées vers le centre du tambour. En tout, le type A produit l'impression d'une boue de lait pasteurisé, tandis que le type $\mathrm{B}$ donne une composition analogue à celle de la boue de lait cru des fermes. Voici l'explication : le poids spécifique des leucocytes est moindre que celui de la caséine et c'est pourquoi la easéine pénètre beaucoup plus avant vers les parois.

Toutes les expériences se réduisent, en fin de compte, au schéma ci-après :

1. Par l'analyse de 23 échantillons, on 'a trouvé :

\begin{tabular}{|c|c|c|c|c|}
\hline Eau & $\begin{array}{l}\text { Matière } \\
\text { sèche }\end{array}$ & $\begin{array}{l}\text { Matières } \\
\text { azotées } \\
\text { dans la } \\
\text { matière } \\
\text { sèche }\end{array}$ & $\begin{array}{l}\text { Caséine dans } \\
\text { la matière } \\
\text { sèche. }\end{array}$ & $\begin{array}{l}\text { Les autres } \\
\text { matières } \\
\text { azotées dans } \\
\text { la matière } \\
\text { sèche }\end{array}$ \\
\hline Moyenne: 65,09 & & 78,97 & 36,20 & 40,04 \\
\hline Minimum : 56,49 & 25,42 & 69,83 & 15,48 & 14,71 \\
\hline Maximum : 74,58 & 43,51 & 91,38 & 64,40 & 59,11 \\
\hline
\end{tabular}

2. La provenance des boues d'écrémeuse joue un rôle capital dans l'aspect extérieur, dans l'humidité et dans l'odeur.

3. La eomposition des matières azotées oscille sensiblement et est influencée, en dehors des circonstances physiologiques, à la fois par la saison, par la composition chimique du lait, par la pasteurisation du lait et par la situation de la boue dans l'écrémeuse.

\title{
LA RÉACTION DE STORCH (1)
}

par le Professeur ORLA-JENSEN et M. O. WINTHER, Vétérinaire

(Travail exécuté au La boratoire de biochimie appliquée de l'Eeole polytechnique de Copenhague.)

Le Professeur Thomé et le Laboratoire ALfred Jorgensen ont donné des observations concordantes sur le phénomène suivant : le lait chauffé à une température telle qu'il donne une réaction de Storch négative immédiatement après le chauffage peut tout de même donner une réaction positive après un certain temps. Les interprétations les plus vraisemblables de ce phénomène sont les suivantes : ou bien la peroxydase est reformée par les bactéries, ou bien cette enzyme n'a été détruite par le chauffage que dans le sérum du lait, mais pas dans les globules blanes (leucocytes). C'est de ceux-ci qu'elle sortirait petit à petit lors du repos du lait.

(1) Molkeritidende, $\mathrm{n}^{\circ} 23,1934$ (traduction C. Wolf). 
L'étude d'OrLa-Jensen ayant pour titre "De l'origine des oxydases et réductases du lait de vache" (1) a prouvé qu'il existe réellement des bactéries qui peuvent produire de l'oxydase dans le lait. On ne peut obtenir une réaction nette de peroxydaxe ressemblant à celle donnée par le lait brut qu'avec des bactéries dénitrifiantes, et cela seulement après plusieurs jours de délai. Puisqu'il ressort des recherches ci-dessus indiquées que du lait donnant une réaction de Storch négative peut donner une réaction de Storch positive après seulement quelques heures de repos, on ne peut guère chercher l'explication de ce phénomène dans l'activité bactérienne. L'autre explication est par contre d'autant plus vraisemblable, comme Barthel l'a montré, que la boue de centrifuge, qui se compose en grande partie de leucocytes, est éminemment riche en peroxydase (2), et comme Mile E. JACOBSEN l'a constaté dans notre laboratoire, il faut des températures bien plus élevées pour détruire la peroxydase (dans les tissus végétaux) aussi longtemps qu'elle est contenue dans les cellules, que quand elle est libre en solution. Dans la grande étude d'OrLa-JEnsen (3), sur la réaction crémométrique, il est aussi indiqué que le lait, qui donnait une réaction de Storch négative à la suite d'un chauffage momentané à $80^{\circ} \mathrm{C}$., donnait une réaction de Storch positive après le même traitement si on l'additionnait d'un peu de boue de centrifuge avant le chauffage.

Dans l'étude d'OrLa-Jensen sur les modifications que le lait de vache subit par le chauffage (4), il est constaté que la peroxydase du lait n'est pas, comme le supposait STORCH, détruite à une température tout à fait précise, mais que là, comme dans toutes les autres transformations du lait, la durée et la température peuvent, dans certaines limites, se remplacer mutuellement. Ainsi, il a été prouvé que la peroxydase était détruite par 5 minutes de chauffage à $75^{\circ} \mathrm{C}$. ou par 30 minutes de chauffage à $72^{\circ} 5 \mathrm{C}$., et il a été prouvé aussi que, même à $70^{\circ} \mathrm{C}$., il était possible de rendre dans un lait la réaction de Storch négative, mais que pour cela il ne fallait pas moins de 5 heures de chauffage. Ces recherches ont été ensuite confirmées par VAN DAM.

Il y a un point qui, pourtant, est resté constamment un peu obscur, c'est la dénomination : chauffage momentané. Du travail original de Sтовон (5), il ne ressort pas clairement comment il obtenait le chauffage momentané. On sait toutefois que la réaction, aussi longtemps qu'on utilisait les anciens appareils de pasteurisa-

(1) C. R. de l'Académie Royale danoise des Sciences, n० 5, 1906.

(2) Milchzeitung, p. 487, 1899.

(3) Le Lait, 1929, p. 1041.

(4) Annuaire agricole de la Suisse, 1905.

(5) $40^{\circ}$ Rapport du Laboratoire d'Essais agricole de Danemark, 1898. 
tion, dans lesquels la durée du chauffage était souvent de quelques. minutes, changeait subitement quand les températures étaient très voisines de $80^{\circ} \mathrm{C}$. Il en fut autrement dès qu'on utilisa les appareils régénérateurs dans lesquels la durée du chauffage est beaucoup plus courte. On s'aperçut très rapidement qu'il fallait élever la température si on voulait être sûr d'avoir un lait ayant une réaction de Storch négative.

VAN DAM, dont, malheureusement, nous ne connaissons le travail que par le compte rendu qu'en a fait WEIGMANN (1), affirme, en effet, que la peroxydase du lait est déjà détruite après 2 secondes 1/2 de chauffage à $80^{\circ} \mathrm{C}$. Ceci n'est pas d'aceord avee nos recherches. La difficulté dans les recherehes de cette sorte est d'arriver rapidement aux températures de $75^{\circ}-79^{\circ} \mathrm{C}$, qui commencent à abîmer la peroxydase, même par un chauffage de courte durée. Pour obtenir ce résultat, le lait fut réparti en tubes minces, qui n'étaient toutefois pas tellement minces pour ne pas pouvoir contenir un thermomètre, mince aussi, qui, bien entendu, était adapté au but cherché. On les préchauffait dans un bain-marie spécial à $70^{\circ} \mathrm{C}$., température qui, comme nous l'avons indiqué, ne détruit la peroxydase qu'après un chauffage prolongé. Les porte-tubes avec les tubes de lait étaient ensuite transportés dans un autre bain-marie qui était maintenu à $2^{\circ}$ ou $8^{\circ}$ au-dessus de la température qu'on désirait obtenir pour le lait. A l'instant même où cette température était atteinte, on plaçait les tubes de lait dans de l'eau courante froide et, après refroidissement, leur contenu était mélangé dans un ballon, de façon à obtenir un échantillon moyen du lait de tous les tubes, qui quoique ce soit peu probable, avaient peut-être été chauffés un peu différemment. Le temps nécessaire pour chauffer le lait de $70^{\circ} \mathrm{C}$. à la température finale fut, lorsque la température du second bainmarie était de $2^{\circ}$ plus élevée, d'environ 50 secondes, et quand la température du second bain-marie était de $8^{\circ} \mathrm{C}$. plus élevée, d'environ 15 secondes. Nous constatames, de cette facon, que quand la. durée du chauffage n'était que de 45-52 secondes, la peroxydase du lait ne commençait à se détruire qu'à $84^{\circ} \mathrm{C}$., et si la durée du chauffage était raccourcie à 15 secondes, le lait pouvait encore avoir une réaction de Storch positive, même s'il était chauffé à $86^{\circ} C$. Avec ces courtes durées de chauffage et aussi de temps à autre, nous avons constaté que le lait qui donnait une réaction de Storch négative immédiatement après le chauffage, donnait une, réaction positive après un certain temps. Dans aucun de ces cas, le lait ne donna jamais une réaction de Storch absolument négative immédiatement après le ohauffage, mais montra après $1 / 2$ à 1 minute une coloration faible. Ce phénomène a surtout lieu si on enrichit spécialement le lait avant le

(1) Frimich mann-Weigmann ; Lehrbuch der Milchwirtschaft, 1932, p. 126. 
chauffage, avec des leucocytes par addition de boue de centrifuge. Nous en donnons, dans le tableau qui suit, un exemple caractéristique. La durée du chauffage avait été d'environ 50 secondes, et le lait, qui n'avait été chauffé qu'à $82^{\circ} \mathrm{C}$., donnait, par conséquent, une réaction de Storch positive stable, ce que nous indiquons par le signe + , tandis que la réaction de Storeh négative (complète aussi $1 / 2$ minute après addition des réactifs) est indiquée par le signe -.

Quoique l'importance des leucocytes pour la réaction de Storch ressorte très nettement de ce tableau, on peut pourtant insister sur le fait que les petites variations qui peuvent normalement exister

\begin{tabular}{|c|c|c|c|c|c|c|}
\hline Pourcen- & & & Temp & ature de & chauffage & \\
\hline $\begin{array}{l}\text { boue de } \\
\text { centri- } \\
\text { fuge ajou- } \\
\text { tée à du } \\
\text { lait pour } \\
\text { nourris- } \\
\text { sons }\end{array}$ & $\begin{array}{c}\text { Temps écoulé } \\
\text { après le } \\
\text { chauffage }\end{array}$ & $\begin{array}{l}82^{\circ} \\
\text { C. }\end{array}$ & $84^{\circ} \mathrm{C}$. & $86^{\circ} \mathrm{C}$. & $88^{\circ} \mathrm{C}$. & $\begin{array}{l}90^{\circ} \\
\text { C. }\end{array}$ \\
\hline 0 & $\begin{array}{l}\text { Immédiatement } \\
3 \text { heures } \\
21 \text { heures }\end{array}$ & $\begin{array}{l}+ \\
+\end{array}$ & $\begin{array}{l}\text { légèrement } \\
\text { teinté } \\
\text { gris- } \\
\text { bleuâtre } \\
+\end{array}$ & teinté & $\begin{array}{c}\text { légèrement } \\
\text { teinté }\end{array}$ & - \\
\hline 1 & $\begin{array}{c}\text { Immédiatement } \\
3 \text { heures } \\
21 \text { heures }\end{array}$ & $\begin{array}{l}+ \\
+ \\
+\end{array}$ & $\begin{array}{c}\text { teinté } \\
\text { gris- } \\
\text { bleuâtre } \\
+\end{array}$ & - & $\begin{array}{l}- \\
\text { teinté }\end{array}$ & $\begin{array}{l}- \\
- \\
-\end{array}$ \\
\hline $21 / 2$ & $\begin{array}{c}\text { Immédiatement } \\
3 \text { heures } \\
21 \text { heures }\end{array}$ & $\begin{array}{l}+ \\
+ \\
+\end{array}$ & $\begin{array}{c}\text { teinté } \\
\text { gris- } \\
\text { bleuâtre } \\
+\end{array}$ & teinté & teinté & $\begin{array}{l}- \\
- \\
-\end{array}$ \\
\hline 5 & $\begin{array}{l}\text { Immédiatement } \\
3 \text { heures } \\
21 \text { heures }\end{array}$ & $\begin{array}{l}+ \\
+\end{array}$ & $\begin{array}{l}\text { gris- } \\
\text { bleuâtre, } \\
\text { gris- } \\
\text { bleuâtre } \\
+\end{array}$ & $\begin{array}{c}\text { teinté } \\
\text { teinté } \\
+\end{array}$ & $\begin{array}{l}\text { teinté } \\
\text { teinté } \\
+\end{array}$ & $\begin{array}{c}- \\
\text { teinté }\end{array}$ \\
\hline
\end{tabular}

dans la teneur en leucocytes du lait, ne semblent pas avoir d'in fluence ici. Du lait de vaches ayant une mammite légère n'a pas du tout montré une teneur spécialement forte en peroxydase. Avec les 
réactifs de Storch, un tel lait a toujours une teinte un peu plus rougeâtre que le lait normal. Nous a vons aussi remarqué que quand du lait avait été chauffé tellement rapidement qu'il y avait possibilité qu'il donne une réaction de Storch positive après repos, ce n'était pas le lait chauffé le plus bas qui donnait la teinte la plus forte. Il est probable que les leucoeytes abandonnent plus facilement la peroxydase non encore détruite, quand ils sont eux-mêmes très touchés par le chauffage.

Comme ce n'est que le chauffage très court qui peut causer des difficultés lors du contrôle de la pasteurisation, le mieux serait de munir tous les systèmes régénérateurs d'un chambreur, et pour ceci, milite également, le fait suivant : si le chauffage est si court qu'il ne peut pas détruire la peroxydase incluse dans les leucocytes, on peut craindre que les bactéries de la tubereulose qui sont incluses dans la boue et les débris de tissu, ne soient également pas détruites, et c'est pourtant là le but de la pasteurisation. C'est pourquoi on peut considérer qu'il est raisonnable d'exiger que le lait pasteurisé par pasteurisation haute donne aussi après un certain repos une réaction de Storch négative. En ce qui concerne le lait destiné à la consommation, on ne peut, en aucun cas, échapper à cette exigence.

\title{
L'AMÉLIORATION DE LA FABRICATION DE LA CASÉINE
}

\author{
par G. GENIN
}

Ingénieur chimiste E. P. C. I.

Le lait écrémé est le sous-produit le plus important de l'industrie laitière et la caséine est la substance la plus intéressante que l'on extrait de ce lait écrémé. L'extraction de la easéine du lait semble à première vue très simple, mais o'est justement ce manque de complication apparente qui a trompé beaucoup d'expérimentateurs qui ont voulu voir dans la précipitation de la caséine un phénomène extrêmement élémentaire. La précipitation de la caséine dans le lait écrémé s'effectue industriellement soit par addition d'acide, soit par fermentation naturelle donnant naissance aux acides nécessaires pour la coagulation de la caséine (nous mettons volontairement de côté la précipitation par la présure).

Il est si facile de laisser surir le lait ou de lui ajouter la quantité d'acide juste nécessaire pour entraîner la précipitation de la caséine, qu'on oublie très fréquemment la complexité chimique et physique de la substance que l'on cherche à obtenir et les variations possibles que peut subir la qualité de ee produit suivant les conditions de sa préparation. Ce sont pourtant ces variations dans les caractéristiques 